\title{
Effect of parameter mismatch on the synchronization of chaotic semiconductor lasers with electro-optical feedback
}

\author{
Y. Chembo Kouomou* and Pere Colet \\ Instituto Mediterráneo de Estudios Avanzados IMEDEA (CSIC-UIB), Campus Universitat de les Illes Balears, \\ E-07122 Palma de Mallorca, Spain \\ Nicolas Gastaud and Laurent Larger \\ GTL-CNRS Telecom, UMR FEMTO-ST 6174, 2-3 rue Marconi, 57070 Metz Cedex, France \\ (Received 6 October 2003; revised manuscript received 19 February 2004; published 28 May 2004)
}

\begin{abstract}
We investigate the detrimental effects of parameter mismatch on the synchronization of semiconductor lasers with electro-optical feedback, whose intensity dynamics can display a hyperchaotic behavior. Analytical techniques are developed to study the statistical properties of the synchronization error as a function of the various types of mismatches. The multiple mismatch case, which is of high experimental interest, is also studied and some compensation conditions allowing the improvement of the synchronization are derived. The analytical predictions are confirmed by numerical simulations and by some experimental measurements.
\end{abstract}

DOI: 10.1103/PhysRevE.69.056226

PACS number(s): 05.45.Vx, 05.45.Xt, 05.45.Jn, 42.65.Sf

\section{INTRODUCTION}

Chaos encryption of data for optical communications is nowadays a widely investigated topic [1-3]. This encryption technique basically relies on the synchronization of two semiconductor lasers operating in a chaotic regime, and is therefore performed at the physical layer of the network architecture. For practical implementations of these communication schemes, it is crucial to achieve a very stable and accurate synchronization between the transmitter and receiver chaotic systems [4]. Particularly important is the double role of the mismatch between characteristics of both systems, which is unavoidable in the fabrication process. On one hand, mismatch can degrade the synchronization quality between the emitter and the receiver. Therefore, synchronization should be robust enough to allow for the use of slightly different devices. On the other hand, the lack of synchronization due to mismatch is a key element for security since it prevents the use by an eventual eavesdropper of a similar device to decode the message.

Despite its importance for practical applications, studies on the effect of parameter mismatch in chaotic synchronization are quite scarce in the literature. In Refs. [5-7], the effect of parameter mismatch was studied analytically for nondelayed low-dimensional chaotic systems. In highdimensional chaotic systems, such as semiconductor lasers with optical feedback, there are some numerical [8] and analytical [9] results. In delayed optoelectronic wavelength oscillators the root-mean-square synchronization error amplitude induced by two simultaneous mismatches has been studied [10]. This is particularly important because successful decryption is not possible when the amplitude of the encrypted message is smaller than the one of the residual oscillations resulting from the parameter mismatch, the socalled "mismatch noise."

\footnotetext{
*Corresponding author: Email address: ckyanne@imedea.uib.es
}

Parameter mismatch is also relevant for chaos-shift keying (CSK) schemes for encryption of binary signals in a chaotic carrier [3]. Effectively, CSK relies on a sequential switching of a given parameter between two values, the first one being matched with the receiver (thereby ensuring synchronization), the second being slightly detuned from the first one (so that the receiver desynchronizes). The synchronization-desynchronization sequence at the receiver enables the message decryption provided that intrinsic mismatch noise allows the distinction between both states. Within that frame, Ref. [11] focused on the performance comparison of the CSK encryption scheme for various "switched" mismatched parameters.

The purpose of the present paper is to study from the analytical, numerical, and experimental points of view the parameter mismatch effects in the synchronization of electrooptical laser chaos. We will first investigate the effects of the various mismatches taken separately (i.e., when one of them is considered, all the others are set to zero), and after that we will study the case of simultaneous parameter mismatches. For the latter case, we will focus on how different parameter mismatches can be compensated to improve the quality of the synchronization. This is of particular relevance since typically some of the mismatches are very difficult to control in practice while others are easily tunable. We will also verify the validity of our analytical results through numerical simulation and some experimental measurements.

The paper is organized as follows. In Sec. II, we present the experimental setup and the dynamical model of the system under study. Sec. III deals with the analysis of the various mismatch effects when they are taken individually, while Sec. IV focuses on multiple mismatches and mismatch compensation. Some experimental results are presented and discussed in Sec. V. Finally, Sec. VI is devoted to concluding remarks.

\section{THE SYSTEM}

The system under study corresponds to the experimental setup represented in Fig. 1. The chaotic transmitter consists 


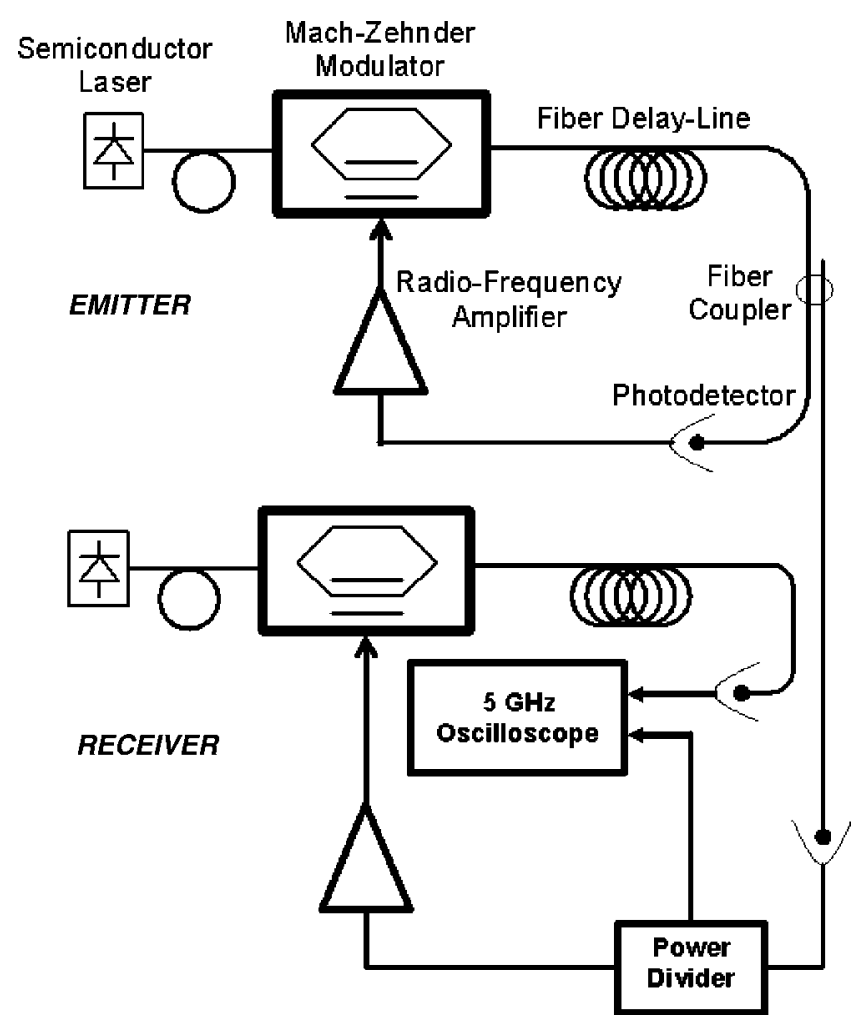

FIG. 1. The experimental setup.

of a closed-loop electro-optical feedback with the following main components: a Mach-Zehnder modulator (of half-wave voltage $V_{\pi}$ and bias voltage $V_{B}$ ) illuminated at $1550 \mathrm{~nm}$ by a continuous-wave semiconductor laser source of power $P$, an optical-fiber delay line of delay time $T$, a fiber coupler in order to insert the chaotic carrier into the fiber communication channel with transmission coefficient $\alpha$, a photodiode with gain $g$ to convert the optical feedback signal into an electrical one, and a radio-frequency amplifier with gain $G$ to drive the Mach-Zehnder modulation electrode. The overall attenuation of this feedback loop (delay line, connectors, etc.) is described in terms of the parameter $\gamma$. The electrooptic modulator is driven by a voltage larger than its halfwave voltage, and it operates in a highly nonlinear regime [12].

The receiver is built symmetrical to the emitter, with the difference that only the light coming from the emitter enters in the delay loop (open-loop receiver). The electronic bandwidth of the feedback loop is considered, in first approximation, to result from two cascaded linear first-order low-pass and high-pass filters, with high and low cutoff frequencies $f_{H}$ and $f_{L}$, respectively. The emitter-receiver coupling is ensured by a $2 \times 2$ fiber coupler which inserts part of the emitter chaotic output into the transmission channel. The open port of the fiber coupler can be used to add at the end of the emitter delay loop a message which is then mixed with the chaotic dynamics and inserted in the transmission channel.

If we consider the variables $x$ and $y$ as the dimensionless voltages across the electric path in the emitter and receiver feedback loops, respectively, their dynamics can be modeled by the following system of coupled differential-delayed equations [12]:

$$
\begin{gathered}
x+\tau \dot{x}+\frac{1}{\theta} \int_{t_{0}}^{t} x(s) d s=\beta \cos ^{2}[x(t-T)+\phi], \\
y+\tau^{\prime} \dot{y}+\frac{1}{\theta^{\prime}} \int_{t_{0}}^{t} y(s) d s=\beta^{\prime} \cos ^{2}\left[x\left(t-T^{\prime}\right)+\phi^{\prime}\right],
\end{gathered}
$$

where

$$
\begin{gathered}
\beta=\pi \alpha \gamma g G P / 2 V_{\pi}, \quad \phi=\pi V_{B} / 2 V_{\pi}, \\
\theta=1 / 2 \pi f_{L}, \quad \tau=1 / 2 \pi f_{H}, \\
\beta^{\prime}=\pi A \gamma^{\prime} g^{\prime} G^{\prime} P^{\prime} / 2 V_{\pi}^{\prime}, \quad \phi^{\prime}=\pi V_{B}^{\prime} / 2 V_{\pi}^{\prime}, \\
\theta^{\prime}=1 / 2 \pi f_{L}^{\prime}, \quad \tau^{\prime}=1 / 2 \pi f_{H}^{\prime} .
\end{gathered}
$$

The receiver parameters are labeled with a prime because they generally differ from the transmitter ones. All additional attenuations in the receiver feedback loop are contained in the coefficient $A$. For simplicity we have neglected the transmission delay time between emitter and receiver. Therefore, we have five control parameters in our model: the delay time $T$, the nonlinear feedback strength $\beta$, the off-set phase $\phi$, the low cutoff response time $\theta$, and the high cutoff response time $\tau$.

To derive analytical predictions, it is convenient to find an approximate stationary integral form for these equations. This has two advantages: first, it skips away the transient dynamics which is incompatible with the statistical analysis and, second, it gives a useful pseudoexplicit mathematical expression for the chaotic dynamic variables [5,6]. The first step of this mathematical transformation is to introduce the variable $u$ as

$$
u(t)=\int_{t_{0}}^{t} x(s) d s
$$

so that Eq. (1) for the emitter dynamics can be formally written as a linear second-order ordinary differential equation for $u$ with a chaotic external forcing

$$
\dot{u}+\tau \ddot{u}+\frac{1}{\theta} u=\beta \cos ^{2}[x(t-T)+\phi] .
$$

The roots of the characteristic polynomial corresponding to the homogeneous solution are

$$
r_{ \pm}=\frac{1}{2 \tau}\left[-1 \pm \sqrt{1-4 \frac{\tau}{\theta}}\right]
$$

Owing to the very large bandwidth of the filter, typically $\tau / \theta \sim 10^{-5} \ll 1$, the roots can be approximated as

$$
r_{+} \simeq-1 / \theta, \quad r_{-} \simeq-1 / \tau \text {. }
$$

They can, respectively, stand for the low and high cutoff angular frequencies of the band-pass filter. Using Eq. (7) the stationary solution for $u$ can be formally expressed as 


$$
u(t)=\beta \int_{t_{0}}^{t}\left[e^{(s-t) / \theta}-e^{(s-t) / \tau}\right] \cos ^{2}[x(s-T)+\phi] d s .
$$

Therefore, the stationary solution for $x$ is given by

$$
x(t)=\beta \int_{t_{0}}^{t} U(s, t) \cos ^{2}[x(s-T)+\phi] d s,
$$

where

$$
U(s, t)=\left(\frac{1}{\tau} e^{(s-t) / \tau}-\frac{1}{\theta} e^{(s-t) / \theta}\right)
$$

is an evolution operator which only depends on the filter parameters $\theta$ and $\tau$. Similarly, one can obtain the following integral expression for the receiver:

$$
y(t)=\beta^{\prime} \int_{t_{0}}^{t} U^{\prime}(s, t) \cos ^{2}\left[x\left(s-T^{\prime}\right)+\phi^{\prime}\right] d s,
$$

with $U^{\prime}(s, t)$ being defined as $U(s, t)$ for the receiver parameters $\theta^{\prime}$ and $\tau^{\prime}$.

\section{SINGLE-PARAMETER MISMATCH}

We define for each parameter $p$ the instantaneous synchronization error as

$$
\epsilon_{\Delta p}(t)=y_{p^{\prime}}(t)-x_{p}(t),
$$

where $\Delta p=p^{\prime}-p$ is the parameter mismatch and $x_{p}(t)$ and $y_{p^{\prime}}(t)$ are the transmitter and receiver time traces obtained with the values of the parameter $p$ and $p^{\prime}$, respectively. We will use two indicators to characterize the effect of the parameter mismatch.

The first one is the normalized root-mean-squared synchronization error which is a quantitative indicator measuring the time-averaged proximity of the emitter and receiver time traces

$$
\sigma_{\Delta p}=\sqrt{\frac{\left\langle\epsilon_{\Delta p}^{2}\right\rangle}{\left\langle x_{p}^{2}\right\rangle}},
$$

where \langle\rangle stands for the time average. The evaluation of this average should be performed integrating over a time much longer than any characteristic time scales of the model, in particular longer than $\theta$, which is the slowest time scale. Furthermore, the integration time should be long enough so that the average reaches a stationary value, which typically takes place when the trajectory samples all the regions of the chaotic attractor. $\sigma$ can be used as an indicator of the sensitivity as well as an indicator of threshold under which any encoded message cannot be recovered, namely, the minimum modulation index.

The second indicator is the normalized cross-correlation function, defined as

$$
\Gamma_{\Delta p}(s)=\frac{\left\langle\left[x_{p}(t)-\left\langle x_{p}(t)\right\rangle\right]\left[y_{p^{\prime}}(t+s)-\left\langle y_{p^{\prime}}(t)\right\rangle\right]\right\rangle}{\sqrt{\left\langle\left[x_{p}(t)-\left\langle x_{p}(t)\right\rangle\right]^{2}\right\rangle\left\langle\left[y_{p^{\prime}}(t)-\left\langle y_{p^{\prime}}(t)\right\rangle\right]^{2}\right\rangle}},
$$

which is a qualitative indicator showing how the slave trajectory is topologically distorted by the mismatch. It may also be considered as a robustness indicator. We should notice that the presence of the low cutoff integral term in Eqs. (1) and (2) implies that the mean value of $x(t)$ and $y(t)$ is zero (otherwise the solutions would linearly diverge to infinity). Therefore for our system Eq. (14) is equivalent to

$$
\Gamma_{\Delta p}(s)=\frac{\left\langle x_{p}(t) y_{p^{\prime}}(t+s)\right\rangle}{\sqrt{\left\langle x_{p}^{2}(t)\right\rangle\left\langle y_{p^{\prime}}^{2}(t)\right\rangle}} .
$$

In the following sections we address the effect of a mismatch in each of the system parameters individually.

\section{A. Delay-time mismatch $(T)$}

The delay mismatch is met experimentally for a different delay length of fiber between the emitter and receiver setup. The global delay includes also the group delay of the rf components and also the eventual electric cables that might be required in the setup.

Assuming $\beta=\beta^{\prime}, \phi=\phi^{\prime}, \theta=\theta^{\prime}$, and $\tau=\tau^{\prime}$ and defining $\Delta T=T^{\prime}-T$, Eq. (11) can be written as

$$
\begin{aligned}
y(t)= & \beta \int_{t_{0}}^{t} U(s, t) \cos ^{2}[x(s-T-\Delta T)+\phi] d s=x(t-\Delta T) \\
& +\beta \int_{t_{0}-\Delta T}^{t_{0}} U\left(s^{\prime}, t-\Delta T\right) \cos ^{2}\left[x\left(s^{\prime}-T\right)+\phi\right] d s^{\prime},
\end{aligned}
$$

where $s^{\prime}=s-\Delta T$. Since the second integral term on the righthand side exponentially decays to zero, one may simply expect in the stationary regime

$$
y(t)=x(t-\Delta T) .
$$

The effect of the $T$ mismatch is to shift the slave time trace back or forth depending on the sign and amplitude of $\Delta T$. This comes from the fact that the receiver equation is a linear differential equation externally forced by a nonlinear function of the chaotic variable $x(t-T)$. Hence, the $T$ mismatch is a change of the time origin, which therefore does not qualitatively affect the dynamics of the slave system. This is clearly illustrated in Fig. 2 which shows the emitter and receiver time traces for different values of $\Delta T$. The time traces have been obtained integrating numerically Eqs. (1) and (2) using a fourth-order predictor-corrector algorithm and a time step of $10 \mathrm{ps}$. The time trace is shown after integrating for a time $t_{0}=10 \theta$, large enough to ensure the decay of the transient dynamics.

An analytic approximation for $\sigma_{\Delta T}$ and $\Gamma_{\Delta T}$ can be obtained as follows. First, we consider the Fourier transform $X(\omega)$ of $x(t)$,

$$
X(\omega)=\frac{1}{\sqrt{2 \pi}} \int_{-\infty}^{+\infty} x(t) e^{-i \omega t} d t,
$$

which satisfies $X(\omega)=X^{*}(-\omega)$ since it is the Fourier transform of a real variable. From Eq. (17) we have $Y(\omega)$ $=e^{-i \omega \Delta T} X(\omega)$, where $Y(\omega)$ is the Fourier transform of $y(t)$. Therefore in Fourier space, the synchronization error is 

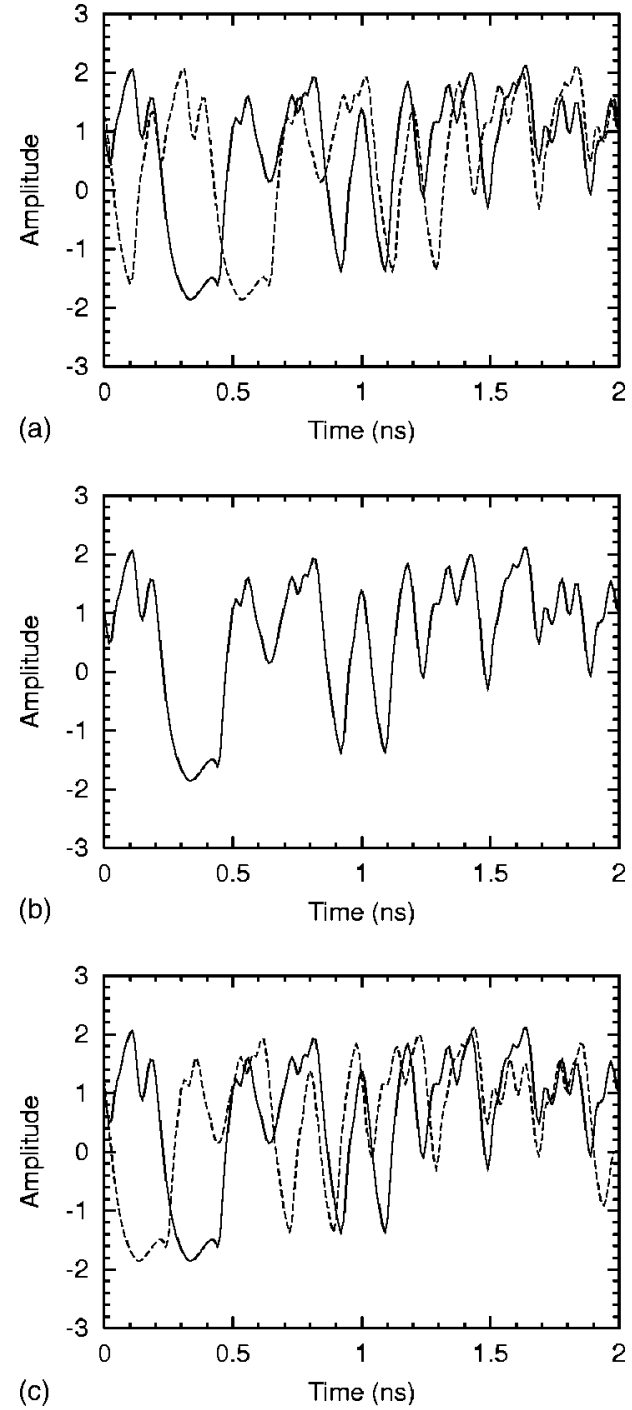

FIG. 2. Transmitter (solid line) and receiver (dashed line) chaotic time traces. We have considered $T=20 \mathrm{~ns}, \beta=5, \phi=0.1, \theta$ $=2 \mu \mathrm{s}$, and $\tau=50 \mathrm{ps}$. These values will be used for all the numerical simulations throughout the whole paper. (a) (top) $\Delta T=0.2 \mathrm{ns,}$ the slave is delayed relatively to the master; (b) (center) $\Delta T=0$, the slave is isochronous to the master; (c) (bottom) $\Delta T=-0.2 \mathrm{~ns}$, the slave anticipates the master. The same initial conditions have been considered for the three cases.

$$
E(\omega)=\left[e^{-i \omega \Delta T}-1\right] X(\omega)=H_{T}(\omega) X(\omega),
$$

where $H_{T}(\omega)$ is the transfer function from $E(\omega)$ to $X(\omega)$ for a $\Delta T$ mismatch. Hence, the synchronization error can theoretically be derived through the Parseval theorem as

$$
\sigma_{\Delta T}^{2}=\frac{\int_{-\infty}^{+\infty}\left|H_{T}(\omega)\right|^{2}|X(\omega)|^{2} d \omega}{\int_{-\infty}^{+\infty}|X(\omega)|^{2} d \omega} .
$$

As the integrals depend on the chaotic time trace they cannot be explicitly determined analytically. However, in first approximation we can consider that $X(\omega)$ is a perfectly flat band-limited white-noise spectrum, the limits being those of the band-pass filter, so that

$$
X(\omega)= \begin{cases}S^{*} & \text { if } \omega \in\left[-\frac{1}{\tau},-\frac{1}{\theta}\right] \\ S & \text { if } \omega \in\left[\frac{1}{\theta}, \frac{1}{\tau}\right] \\ 0 & \text { otherwise, }\end{cases}
$$

where $|S|$ is arbitrary (the value of $|S|$ is not relevant here because the synchronization error is normalized with the average intensity of the chaotic carrier). We are therefore led to the following expression for the synchronization error:

$$
\sigma_{\Delta T}^{2} \approx \frac{1}{\tau^{-1}-\theta^{-1}} \int_{1 / \theta}^{1 / \tau}\left|H_{T}(\omega)\right|^{2} d \omega \approx 2\left[1-\operatorname{sinc}\left(\frac{\Delta T}{\tau}\right)\right],
$$

where sinc is the sine-cardinal function. The accuracy of this result relies on the validity of the approximation when considering that the chaotic spectrum looks like a white noise within a bandwidth defined by the cutoff times $\theta$ and $\tau$.

Figure 3(a) displays the comparison between numerical and analytical results for the synchronization error. Despite the simplicity of the approximation, Eq. (22) gives a good prediction although the numerical results show for small mismatch values a larger synchronization error than theoretically predicted. Both analytical prediction and numerical results indicate a very high sensitivity to time-delay mismatch: a $1 \%$ synchronization error is induced when $\Delta T=1 \mathrm{ps}$, that is, for a relative error of $\Delta T / T \simeq 5 \times 10^{-5}$. As indicated by Eq. (22), this high sensitivity comes from the large bandwidth $\Delta f \simeq 1 / 2 \pi \tau \gg 1 / T$. Consequently, for a satisfying synchronization quality, the length of the fiber delay lines should practically be adjusted with a relative precision of the order of $10^{-6}$. In the case of a larger bandwidth filter (as it would ideally be the case), this sensitivity would be increased proportionally to the high cutoff frequency.

To determine analytically the cross-correlation function, we can use a corollary of the Wiener-Khintchin theorem which states that the cross-correlation function is the inverse Fourier transform of the cross-power spectral density function [15]. Hence, the expression of $\Gamma_{\Delta T}$ as a function of its lag-time argument $s$ reads

$$
\begin{aligned}
\Gamma_{\Delta T}(s) & =\frac{\int_{-\infty}^{+\infty} X(\omega) Y^{*}(\omega) e^{i \omega s} d \omega}{\sqrt{\left[\int_{-\infty}^{+\infty}|X(\omega)|^{2} d \omega\right]\left[\int_{-\infty}^{+\infty}|Y(\omega)|^{2} d \omega\right]}} \\
& =\operatorname{sinc}\left(\frac{s+\Delta \mathrm{T}}{\tau}\right) .
\end{aligned}
$$

Figure 3(b) displays the comparison between Eq. (23) and the numerical results for the cross correlation at zero lag time $(s=0)$ obtained integrating Eqs. (1) and (2). Again, despite its simplicity, Eq. (23) is a quite good approximation of the correlation function. The numerical calculations show that by 

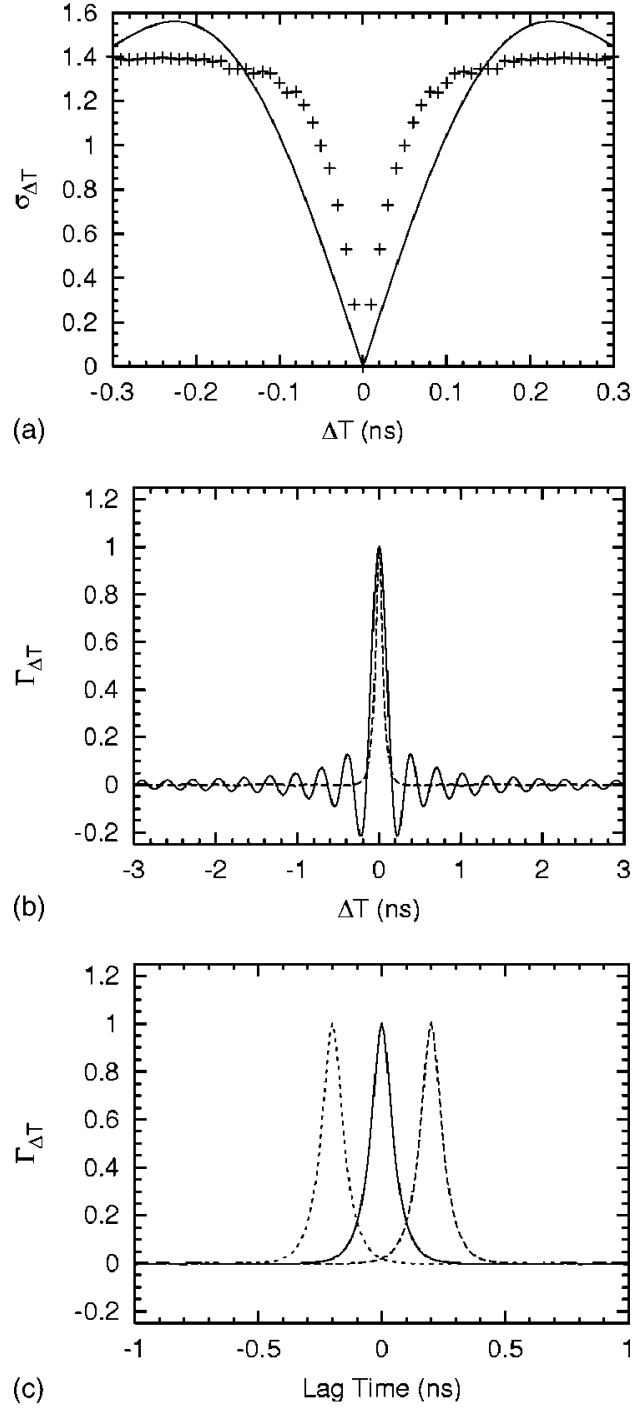

FIG. 3. (a) (top) Average synchronization error and (b) (center) cross correlation function at equal times for time-delay mismatch. The analytical results are displayed in solid lines, while the numerical results obtained from integration of Eqs. (1) and (2) are displayed in symbols or dotted lines. The average synchronization error and the cross-correlation function as, respectively, defined by Eqs. (13) and (15) are dimensionless quantities. (c) (bottom) crosscorrelation function $\Gamma_{\Delta T}(s)$ for $\Delta T=0.2 \mathrm{~ns}$ (long-dashed line), $\Delta T$ $=0$ (continuous line), and $\Delta T=-0.2 \mathrm{~ns}$ (short-dashed line). Notice that it is the same curve, shifted to the right or to the left by the delay time mismatch, $\Delta T$.

increasing the delay time mismatch, the correlation decays, in fact, slightly faster than the analytical prediction (23). The oscillations that appear in the theoretical curve come from the vertical cutoff we have considered for the hyperchaotic spectrum $[X(\omega)$ was approximated as a rectangular function]. They could be eliminated by approximating $X(\omega)$ as a function with a smooth decay to zero, which would be a more realistic approximation for the spectra of the chaotic time trace. However, this would be made at the cost of a higher complexity for the calculations.

Equation (23) also indicates that, as we may expect from Eq. (17), the effect of $\Delta T$ is to shift the autocorrelation func- tion to the left $(\Delta T<0)$ or to the right $(\Delta T>0)$ by an amount $\Delta T$. This analytical prediction is numerically confirmed in Fig. 3(c). The influence of time-delay mismatch is in absolute rather than relative value since the time traces and correlation functions are shifted proportionally to $\Delta T$.

\section{B. Nonlinear feedback-strength mismatch $(\boldsymbol{\beta})$}

The $\beta$ mismatch corresponds to a normalized gain in the experimental setup, which includes various physical parameters: the electronic feedback gain, the electro-optic sensitivity of the modulator $\left(V_{\pi}\right)$, the detector sensitivity, the optical losses, and the cw laser optical power. The precise dependence on these parameters is given in Eq. (3). Only the mismatch in the resulting $\beta$ is relevant and not in the individual physical parameters, since, for example, a rf gain mismatch can be compensated by an optical power mismatch.

Assuming $\phi=\phi^{\prime}, \theta=\theta^{\prime}, \tau=\tau^{\prime}, T=T^{\prime}$, and defining $\Delta \beta$ $=\beta^{\prime}-\beta$ one obtains from Eqs. (9) and (11) that $x(t)$ $=\beta y(t) / \beta^{\prime}$. The receiver time trace is exactly the same as the transmitter but with different amplitude. This can also be obtained (1) directly and (2) by rescaling $y$ with $\beta / \beta^{\prime}$. Therefore, this result is mathematically exact, independent of the approximations we have done to obtain the stationary integral form. Then $\epsilon(t)=(\Delta \beta / \beta) x(t)$, so that the average synchronization error is

$$
\sigma_{\Delta \beta}=\left|\frac{\Delta \beta}{\beta}\right| .
$$

Therefore the synchronization error depends on the relative $\beta$ mismatch. Thus, for example, a $1 \% \Delta \beta$ mismatch induces a $1 \%$ synchronization error. Since $x$ and $y$ are strictly proportional, the cross-correlation function at equal times is always perfect, namely, $\Gamma_{\Delta \beta}(0)=1$.

Figure 4 displays the synchronization error and the cross correlation at equal times. The analytical results coincide exactly with the numerical ones, as expected from the fact that no approximation was needed to obtain the analytical results.

\section{Off-set phase mismatch $(\phi)$}

The $\phi$ mismatch stems from a residual optical path difference in the emitter and receiver Mach-Zehnder modulators, but it can be experimentally compensated while changing the dc bias of the electro-optic voltage.

To obtain an analytical approximation for the effects of this mismatch, we rewrite Eq. (9) as

$$
x(t)=\frac{\beta}{2} \int_{t_{0}}^{t} U(s, t) d s+\frac{\beta}{2} \int_{t_{0}}^{t} U(s, t) \cos [2 x(s-T)+2 \phi] d s .
$$

The first integral term exponentially decays to zero, which physically comes from the dc filtering property of the bandpass filter. Therefore the stationary solution of $x(t)$ and $y(t)$ can be written as 

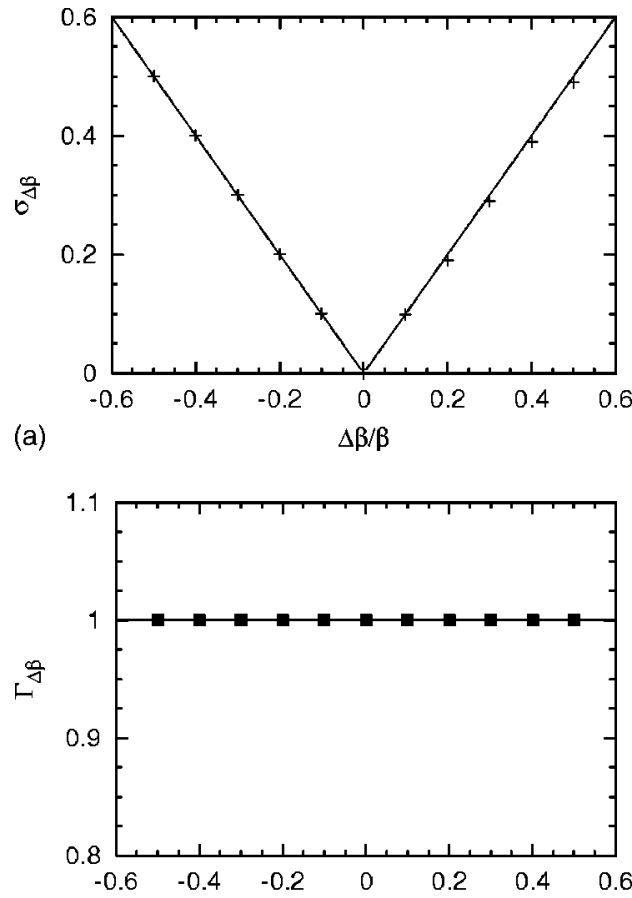

(b)

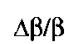

FIG. 4. (a) (top) Average synchronization error and (b) (bottom) cross-correlation at equal times for $\beta$ mismatch. The analytical results are in solid lines and the numerical ones are in symbols.

$$
\begin{gathered}
x(t)=\frac{\beta}{2} \int_{t_{0}}^{t} U(s, t) \cos [2 x(s-T)+2 \phi] d s, \\
y(t)=\frac{\beta}{2} \int_{t_{0}}^{t} U^{\prime}(s, t) \cos \left[2 x\left(s-T^{\prime}\right)+2 \phi^{\prime}\right] d s .
\end{gathered}
$$

For the sake of compactness, let us introduce

$$
Q(s, t, \phi)=U(s, t) \cos [2 x(s-T)+2 \phi] .
$$

Assuming $\beta=\beta^{\prime}, \theta=\theta^{\prime}, \tau=\tau^{\prime}, T=T^{\prime}$, and defining $\Delta \phi=\phi^{\prime}$ $-\phi$, the instantaneous synchronization error may be expressed as

$$
\epsilon(t)=-\beta \sin (\Delta \phi) \int_{t_{0}}^{t} Q\left(s, t, \phi+\frac{\Delta \phi}{2}-\frac{\pi}{4}\right) d s
$$

Starting from here, we assume that for long enough delay times, the average properties of the chaotic attractor are independent of the off-set phase. This statistical phase invariance hypothesis is supported by the results from the numerical computation of the Lyapunov exponents of the system [13], and also by previous investigations led by Ref. [14] which show that when $\beta$ and $T$ are large enough, the number and values of the positive Lyapunov exponents are practically independent of the off-set phase. Therefore

$$
\left\langle\epsilon_{\Delta \phi}^{2}\right\rangle \approx \beta^{2} \sin ^{2}(\Delta \phi)\left\langle\left[\int_{t_{0}}^{t} Q(s, t, \phi) d s\right]^{2}\right\rangle=4 \sin ^{2}(\Delta \phi)\left\langle x^{2}\right\rangle .
$$

Finally, the average synchronization error is given by

$$
\sigma_{\Delta \phi}=2|\sin (\Delta \phi)| \text {. }
$$

$\sigma_{\Delta \phi}$ does not depend on the value of the off-set phase $\phi$ itself, which is a consequence of our assumption, therefore there is no "optimal" value of $\phi$. Equation (30) also indicates the extreme sensitivity of this parameter; for example, if we consider a small $\Delta \phi$ mismatch of 0.01 ( $\phi$ is a ratio between two voltages), the induced synchronization error is $2 \%$, which is quite important.

According to Eq. (26) the numerator of the cross correlation at equal times is given by

$$
\begin{aligned}
\langle x y\rangle= & \frac{\beta^{2}}{4} \cos (2 \Delta \phi)\left\langle\left[\int_{t_{0}}^{t} Q(s, t, \phi) d s\right]^{2}\right\rangle-\frac{\beta^{2}}{4} \sin (2 \Delta \phi) \\
& \times\left\langle\left[\int_{t_{0}}^{t} Q(s, t, \phi) d s\right]\left[\int_{t_{0}}^{t} Q\left(s, t, \phi-\frac{\pi}{4}\right) d s\right]\right\rangle .(31)
\end{aligned}
$$

We here assume that the hyperchaotic behavior induced by the electro-optical oscillator is ergodic, so that we can equate the average over time to an average over the attractor in phase space. Although we cannot prove this hypothesis, it is consistent with the fact that time averages are performed over long times so that they reach a stationary value as discussed after Eq. (13). Furthermore, the results we obtain are in quite good agreement with numerical simulations as we will show below. The ergodic assumption implies that the last average of Eq. (31) vanishes since it involves the product of a symmetric with an antisymmetric function. Therefore

$$
\langle x y\rangle \approx \cos (2 \Delta \phi)\left\langle x^{2}\right\rangle .
$$

We also have

$$
\begin{aligned}
\left\langle y^{2}\right\rangle & =\frac{\beta^{2}}{4}\left\langle\left[\int_{t_{0}}^{t} Q(s, t, \phi+\Delta \phi) d s\right]^{2}\right\rangle \\
& \simeq \frac{\beta^{2}}{4}\left\langle\left[\int_{t_{0}}^{t} Q(s, t, \phi) d s\right]^{2}\right\rangle=\left\langle x^{2}\right\rangle,
\end{aligned}
$$

where we have again assumed that the time-average properties of the chaotic attractor are independent of the off-set phase delay. Therefore the cross correlation is given by

$$
\Gamma_{\Delta \phi}(0)=\cos (2 \Delta \phi) .
$$

Logically it turns out that the cross correlation and the synchronization error are $\pi$ periodic as the nonlinear feedback function. Also from Eq. (34) for $\Delta \phi= \pm \pi / 2, \Gamma_{\phi}(0)=-1$, so that the receiver time trace is in exact phase opposition with respect to the emitter one (that is why the corresponding synchronization error value is exactly 2 in that case).

Figure 5 displays the average synchronization error and the cross correlation at equal times. Equation (34) shows an excellent agreement with the numerical results, which indicates the validity of the assumptions we have made. How- 


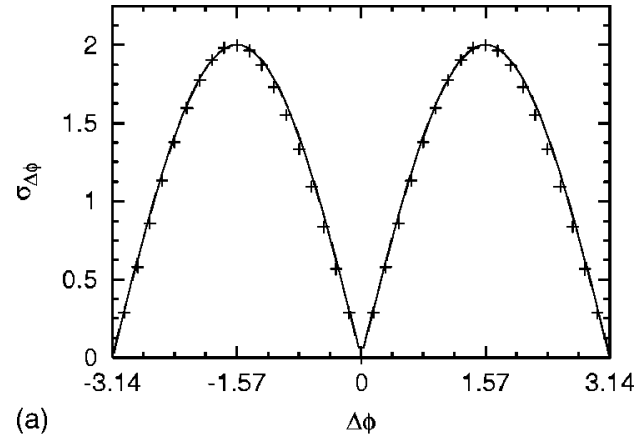

(a)

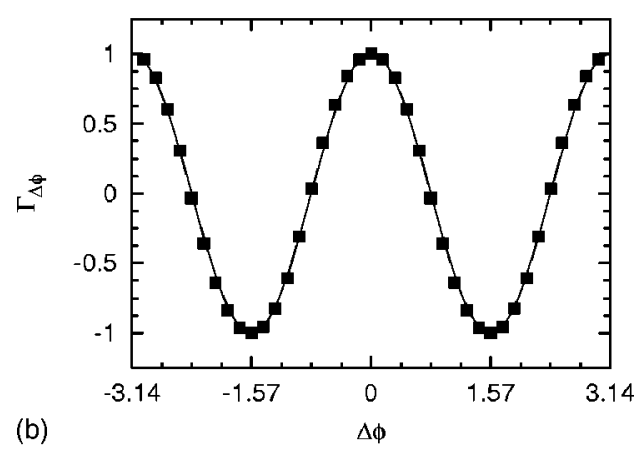

FIG. 5. (a) (top) Average synchronization error and (b) (bottom) cross correlation at equal times for off-set phase mismatch. Analytical approximations are shown in solid lines and numerical results in symbols.

ever, one may not expect such a good concordance in the case of small values of $\beta$ or $T$, where, for example, the statistical off-set phase invariance cannot be guaranteed.

The above results can also be obtained in the Fourier space as follows. From Eq. (26) we have

$$
Y(\omega)=e^{2 i \Delta \phi} V(\omega)+e^{-2 i \Delta \phi} V^{*}(-\omega),
$$

where $V(\omega)$ is defined as

$$
V(\omega)=\frac{\beta}{4 \sqrt{2 \pi}} \int_{-\infty}^{+\infty} \int_{t_{0}}^{t} U(s, t) e^{2 i[x(s-T)+\phi} e^{-i \omega t} d s d t
$$

We can write $V(\omega)=\frac{1}{2}[X(\omega)+i A(\omega)]$, where

$$
A(\omega)=\frac{\beta}{2 \sqrt{2 \pi}} \int_{-\infty}^{+\infty} \int_{t_{0}}^{t} U(s, t) \sin [2 x(s-T)+2 \phi] e^{-i \omega t} d s d t
$$

Then using $X(\omega)=X^{*}(-\omega)$ and $A(\omega)=A^{*}(-\omega)$ (which come from the fact that they are Fourier transforms of real functions),

$$
Y(\omega)=\cos (2 \Delta \phi) X(\omega)-\sin (2 \Delta \phi) A(\omega)
$$

The synchronization error is

$$
E(\omega)=[\cos (2 \Delta \phi)-1] X(\omega)-\sin (2 \Delta \phi) A(\omega) .
$$

The average synchronization error can be obtained through the Parseval theorem

$$
\sigma_{\Delta \phi}^{2}=\frac{\int_{-\infty}^{+\infty}|E(\omega)|^{2} d \omega}{\int_{-\infty}^{+\infty}|X(\omega)|^{2} d \omega}
$$

The numerator can be written as

$$
\begin{aligned}
\int_{-\infty}^{+\infty}|E(\omega)|^{2} d \omega= & {[\cos (2 \Delta \phi)-1]^{2} \int_{-\infty}^{+\infty}|X(\omega)|^{2} d \omega } \\
& +\sin ^{2}(2 \Delta \phi) \int_{-\infty}^{+\infty}|A(\omega)|^{2} d \omega-[\cos (2 \Delta \phi) \\
& -1] \times \sin (2 \Delta \phi) \int_{-\infty}^{+\infty}\left[X(\omega) A^{*}(\omega)\right. \\
& \left.+X^{*}(\omega) A(\omega)\right] d \omega .
\end{aligned}
$$

The integral $\int_{-\infty}^{+\infty} X(\omega) A^{*}(\omega) d \omega$ can be written as

$$
\begin{aligned}
\frac{\beta}{4} \int_{-\infty}^{+\infty} & \int_{-\infty}^{+\infty} \int_{-\infty}^{+\infty}\left[\int_{t_{0}}^{t} U(s, t) \cos [2 x(s-T)+2 \phi] d s\right] \\
\times & {\left[\int_{t_{0}}^{t^{\prime}} U\left(s, t^{\prime}\right) \sin [2 x(s-T)+2 \phi] d s\right] e^{i \omega\left(t^{\prime}-t\right)} d t d t^{\prime} d \omega }
\end{aligned}
$$

which corresponds to the average appearing in the last term of Eq. (31). Therefore the last term of Eq. (41) vanishes. On the other hand, the function $A(\omega)$ obtained from Eq. (37) for an off-set phase $\phi$ is precisely $X(\omega)$ for an off-set phase $\phi$ $-\pi / 4$. Therefore under the assumption that the average properties of the chaotic attractor are independent of the off-set phase, we are going to consider that

$$
\int_{-\infty}^{+\infty} f(\omega)|A(\omega)|^{2} d \omega=\int_{-\infty}^{+\infty} f(\omega)|X(\omega)|^{2} d \omega
$$

for any function $f(\omega)$. Then the average synchronization error is given by

$$
\sigma_{\Delta \phi}^{2}=[\cos (2 \Delta \phi-1)]^{2}+\sin ^{2}(2 \Delta \phi)=4 \sin ^{2}(\Delta \phi),
$$

exactly as obtained before. The cross correlation can be determined as in Sec. III A. We have

$$
\begin{aligned}
\int_{-\infty}^{+\infty} X(\omega) Y^{*}(\omega) e^{i \omega s} d \omega= & \cos (2 \Delta \phi) \int_{-\infty}^{+\infty}|X(\omega)|^{2} e^{i \omega s} d \omega \\
& -\sin (2 \Delta \phi) \int_{-\infty}^{+\infty} X(\omega) A^{*}(\omega) e^{i \omega s} d \omega .
\end{aligned}
$$

Here, and later on in Sec. IV, we have to evaluate integrals of the form $\int_{-\infty}^{+\infty} f(\omega) X(\omega) A^{*}(\omega) d \omega$, where $f(\omega)=f_{\mathrm{R}}(\omega)+i f_{\mathrm{I}}(\omega)$ is a complex function such that the real part $f_{R}(\omega)$ is symmetric in $\omega$ and the imaginary part $f_{I}(\omega)$ is antisymmetric. As discussed above $X(\omega) A^{*}(\omega)$ is an antisymmetric function, therefore the symmetric $f_{R}(\omega)$ does not contribute to the integral. To evaluate the contribution from $f_{I}(\omega)$ we assume the band- 
pass filter approximation of the chaotic spectrum given in Eq. (21), so that

$$
\int_{-\infty}^{+\infty} f(\omega) X(\omega) A^{*}(\omega) d \omega=-2|S|^{2} \int_{1 / \theta}^{1 / \tau} f_{\mathrm{I}}(\omega) d \omega .
$$

Finally we obtain

$$
\Gamma_{\Delta \phi}(s)=\frac{\sin \left(2 \Delta \phi+s \tau^{-1}\right)-\sin \left(2 \Delta \phi+s \theta^{-1}\right)}{s\left(\tau^{-1}-\theta^{-1}\right)},
$$

which, in the limit $s \rightarrow 0$, reduces to Eq. (34). The Fourier approach just discussed will be used later on when discussing the effect of simultaneous mismatch on different parameters.

\section{Low cutoff response time mismatch $(\theta)$}

The low and high cutoff response times are practically determined by the rf amplifier and by the photodiode bandwidth. In practice, it is very difficult to tune them, and the components involved in the setup are ordered to be matched by the suppliers.

Assuming all the other parameters are equal, we have from Eqs. (1) and (2)

$$
\epsilon+\tau \dot{\epsilon}+\frac{1}{\theta+\Delta \theta} \int_{t_{0}}^{t} \epsilon(s) d s=\frac{\Delta \theta}{\theta(\theta+\Delta \theta)} \int_{t_{0}}^{t} x(s) d s,
$$

which in Fourier domain gives

$$
E(\omega)=\frac{\frac{\Delta \theta}{\theta}}{1+i \omega(\theta+\Delta \theta)(1+i \omega \tau)} X(\omega) .
$$

As in Sec. III A, we approximate $X(\omega)$ by a rectangular function, so that

$$
\sigma_{\Delta \theta}^{2}=\frac{\left(\frac{\Delta \theta}{\theta}\right)^{2}}{\frac{1}{\tau}-\frac{1}{\theta}} \int_{1 / \theta}^{1 / \tau} \frac{d \omega}{\left[1-\omega^{2} \tau(\theta+\Delta \theta)\right]^{2}+\omega^{2}(\theta+\Delta \theta)^{2}} .
$$

At first order in $\tau / \theta$, we have

$$
\sigma_{\Delta \theta}=\sqrt{\frac{\tau}{\theta}}\left|\frac{\Delta \theta}{\theta}\right| \sqrt{\frac{\pi-2 \arctan (1+\Delta \theta / \theta)}{2(1+\Delta \theta / \theta)}} .
$$

For typical parameter values, this synchronization error is very small (of the order $10^{-3}$ ) hence, we can conclude that even a large mismatch for $\theta$ does not significantly affect the quality of the synchronization. Physically this comes from the fact that the low cutoff frequency is of the order of tens of kilohertz, while the chaotic oscillations are typically within the gigahertz range. Therefore, any mismatch at such a low frequency range cannot really destabilize the synchronization manifold. Figure 6 displays the average synchronization error. The analytical approximation (50) shows a very good agreement with the numerical results obtained integrating Eqs. (1) and (2) specially for small mismatch. The small
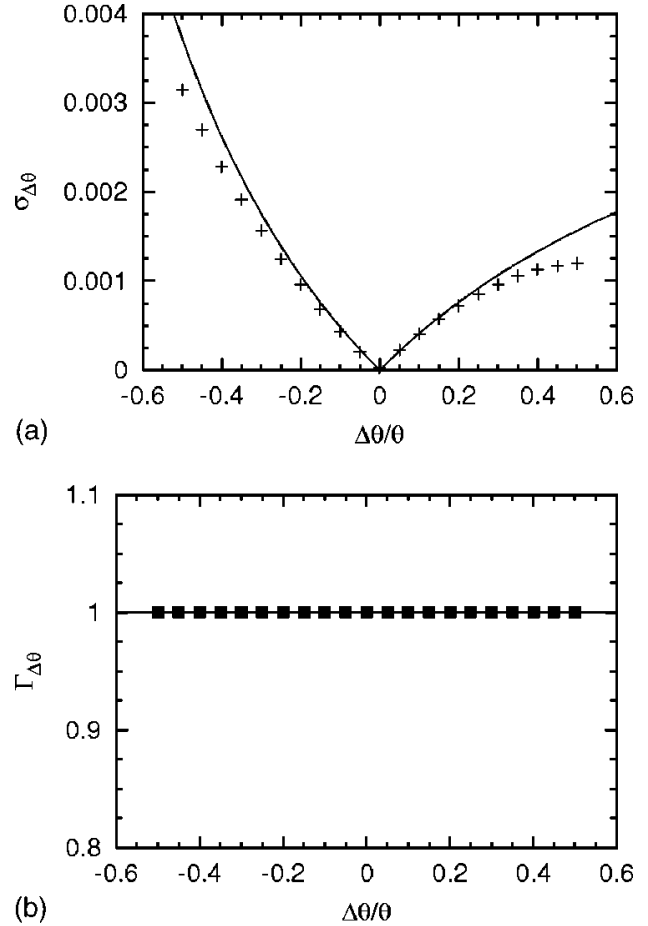

FIG. 6. (a) (top) Average synchronization error and (b) (bottom) cross correlation at equal times for $\theta$ mismatch. Analytical approximations are shown in solid lines and numerical results in symbols.

synchronization error indicates that the emitter and receiver time traces are very similar, therefore we can assume that the equal time cross correlation is practically perfect, yielding $\Gamma_{\Delta \theta}(0)=1$.

\section{E. High cutoff frequency mismatch $(\tau)$}

Assuming all the other parameters are equal, from Eqs. (1) and (2) we have for $\epsilon(t)$,

$$
\epsilon+(\tau+\Delta \tau) \dot{\epsilon}+\frac{1}{\theta} \int_{t_{0}}^{t} \epsilon(s) d s=-\Delta \tau \dot{x}
$$

which in the Fourier domain gives

$$
E(\omega)=\frac{\omega^{2} \theta \Delta \tau}{1-\omega^{2} \theta(\tau+\Delta \tau)+i \omega \theta} X(\omega) .
$$

Approximating $X(\omega)$ by a rectangular function we obtain

$$
\sigma_{\Delta \tau}^{2}=\frac{(\Delta \tau)^{2}}{\frac{1}{\tau}-\frac{1}{\theta}} \int_{1 / \theta}^{1 / \tau} \frac{\omega^{4} \theta^{2}}{\left[1-\omega^{2} \theta(\tau+\Delta \tau)\right]^{2}+\omega^{2} \theta^{2}} d \omega,
$$

so that, at first order in $\tau / \theta$,

$$
\sigma_{\Delta \tau}=\left|\frac{\Delta \tau}{\tau+\Delta \tau}\right| \sqrt{1-\frac{\arctan (1+\Delta \tau / \tau)}{1+\Delta \tau / \tau}} .
$$

The cross correlation can be calculated in a similar way as in Sec. III A. Approximating $X(\omega)$ by a rectangular function, at first order in $\tau / \theta$, we have 

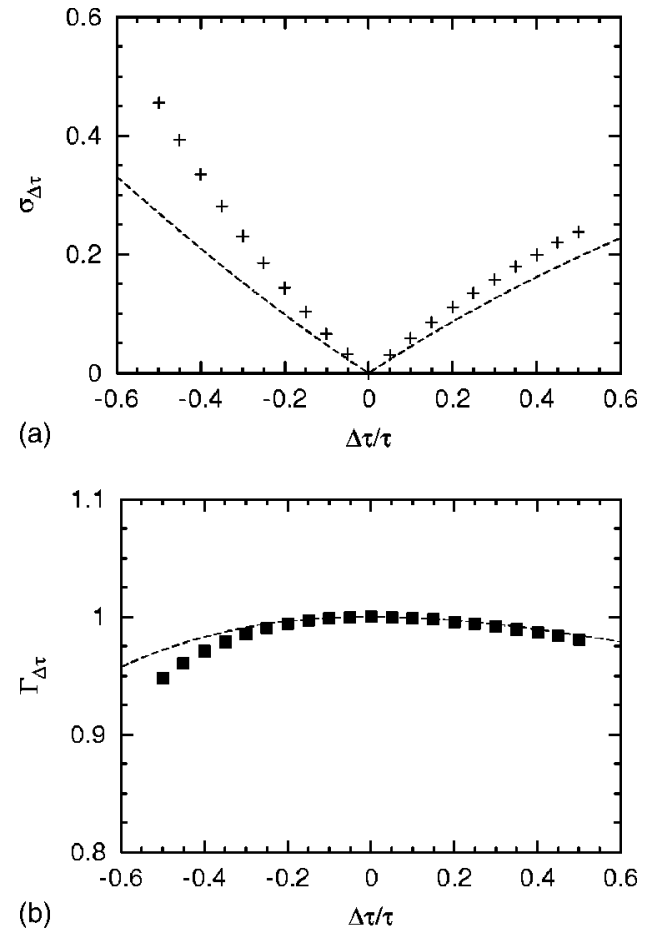

FIG. 7. (a) (top) Average synchronization error and (b) (bottom) cross correlation at equal times for $\tau$ mismatch. Analytical approximations are shown in solid lines and numerical results in symbols.

$$
\Gamma_{\Delta \tau}(0)=\frac{1+\frac{\Delta \tau}{\tau}+\frac{\Delta \tau}{\tau} \arctan \left(1+\frac{\Delta \tau}{\tau}\right)}{\left(1+\frac{\Delta \tau}{\tau}\right) \sqrt{1+\frac{\Delta \tau}{\tau} \arctan \left(1+\frac{\Delta \tau}{\tau}\right)}} .
$$

Equation (54) implies that for typical parameter values there is a relatively high sensitivity of the synchronization manifold to the high cutoff frequency, since a $1 \%$ error in $\tau$ induces approximately a $0.5 \%$ synchronization error.

Figure 7 displays the synchronization error and the cross correlation at equal times. The analytical approximations show a quite satisfactory agreement with the numerical results, mainly for small parameter mismatches.

\section{THE MULTIPLE-PARAMETER MISMATCH CASE}

We now focus on the general and important case where all the mismatches are simultaneously taken into account. This would first enable us to study the synchronization quality in situations that are closer to the experimental ones, and second to understand the interplay between the various mismatches.

We first rewrite Eq. (1) and (2) as

$$
x+\tau \dot{x}+\frac{1}{\theta} \int_{t_{1}}^{t} x(s) d s=\beta \cos [2 x(t-T)+2 \phi]
$$

$$
y+\tau^{\prime} \dot{y}+\frac{1}{\theta^{\prime}} \int_{t_{1}}^{t} y(s) d s=\beta^{\prime} \cos \left[2 x\left(t-T^{\prime}\right)+2 \phi^{\prime}\right],
$$

where $t_{1}$ is such that $\int_{t_{0}}^{t_{1}} x(s) d s=\beta \theta$. We introduce two complex variables $u, v$, such that

$$
\begin{gathered}
v+\tau \dot{v}+\frac{1}{\theta} \int_{t_{1}}^{t} v(s) d s=\beta e^{2 i[x(t-T)+\phi]}, \\
w+\tau^{\prime} \dot{w}+\frac{1}{\theta^{\prime}} \int_{t_{1}}^{t} w(s) d s=\beta^{\prime} e^{2 i\left[x\left(t-T^{\prime}\right)+\phi^{\prime}\right]},
\end{gathered}
$$

so $\operatorname{Re}[v]=x$ and $\operatorname{Re}[w]=y$. Then, in the Fourier space,

$$
\begin{aligned}
& X(\omega)=V(\omega)+V^{*}(-\omega), \\
& Y(\omega)=W(\omega)+W^{*}(-\omega) .
\end{aligned}
$$

We know that the effect of the delay time mismatch in the stationary state is to shift in time the chaotic trajectory. Therefore we introduce $w_{1}(t)=w(t+\Delta t)$. Assuming that in the stationary state $\int_{t_{1}-\Delta T^{t} w_{1}}^{t-\Delta T}(s) d s=\int_{t_{1}}^{t} w_{1}(s) d s$, then

$$
w_{1}+\tau^{\prime} \dot{w}_{1}+\frac{1}{\theta^{\prime}} \int_{t_{1}}^{t} w_{1}(s) d s=\beta^{\prime} e^{2 i\left[x(t-T)+\phi^{\prime}\right]} .
$$

From Eqs. (58) and (61) we have

$$
\begin{aligned}
v+\tau \dot{v}+\frac{1}{\theta} \int_{t_{1}}^{t} v(s) d s= & \frac{\beta}{\beta^{\prime}}\left[w_{1}+\tau^{\prime} \dot{w}_{1}\right. \\
& \left.+\frac{1}{\theta^{\prime}} \int_{t_{1}}^{t} w_{1}(s) d s\right] e^{-2 i \Delta \phi} .
\end{aligned}
$$

Therefore, in the Fourier space $W_{1}(\omega)$ $=\left(\beta^{\prime} / \beta\right) e^{2 i \Delta \phi} F(\omega) V(\omega)$, where

$$
F(\omega)=\frac{\frac{\theta^{\prime}}{\theta}-\omega^{2} \tau \theta^{\prime}+i \omega \theta^{\prime}}{1-\omega^{2} \tau^{\prime} \theta^{\prime}+i \omega \theta^{\prime}} .
$$

From the definition of $w_{1}$ we have $W(\omega)=e^{-i \omega \Delta T} W_{1}(\omega)$, so that

$$
W(\omega)=\frac{\beta^{\prime}}{\beta} e^{-i \omega \Delta T} e^{2 i \Delta \phi} F(\omega) V(\omega)
$$

Then

$$
Y(\omega)=\frac{\beta^{\prime}}{\beta} e^{-i \omega \Delta T} F(\omega)[\cos (2 \Delta \phi) X(\omega)-\sin (2 \Delta \phi) A(\omega)],
$$

where $A(\omega)$ is the Fourier transform of the imaginary part of $v$ and where $X(\omega)=X^{*}(-\omega), \quad A(\omega)=A^{*}(-\omega)$, and $F(\omega)$ $=F^{*}(-\omega)$ have been assumed. Therefore, the synchronization error is given by 


$$
\begin{aligned}
E(\omega)= & \frac{\beta^{\prime}}{\beta} e^{-i \omega \Delta T} F(\omega)[\cos (2 \Delta \phi) X(\omega)-\sin (2 \Delta \phi) A(\omega)] \\
& -X(\omega) .
\end{aligned}
$$

The average synchronization error can be obtained through the Parseval theorem, Eq. (40). Calculating the integrals as discussed in Sec. III C we obtain

$$
\begin{aligned}
\sigma^{2}= & \frac{1}{\tau^{-1}-\theta^{-1}}\left\{\left(1+\frac{\Delta \beta}{\beta}\right)^{2} \int_{1 / \theta}^{1 / \tau}|F(\omega)|^{2} d \omega-2\left(1+\frac{\Delta \beta}{\beta}\right)\right. \\
& \times\left[\cos (2 \Delta \phi) \int_{1 / \theta}^{1 / \tau} \operatorname{Re}\left[e^{-i \omega \Delta T} F(\omega)\right] d \omega\right. \\
& \left.\left.+\sin (2 \Delta \phi) \int_{1 / \theta}^{1 / \tau} \operatorname{Im}\left[e^{-i \omega \Delta T} F(\omega)\right] d \omega\right]\right\} .
\end{aligned}
$$

Therefore, the squared synchronization error is a quadratic function of $\Delta \beta$, and a sinusoidal function of $\Delta \phi$.

Evaluating the integrals up to the second order in parameter mismatch, we obtain the following expression for the synchronization error:

$$
\begin{aligned}
\sigma^{2}= & \frac{1}{3}\left(\frac{\Delta T}{\tau}\right)^{2}+\left(\frac{\Delta \beta}{\beta}\right)^{2}+4(\Delta \phi)^{2}+\left(1-\frac{\pi}{4}\right)\left(\frac{\Delta \tau}{\tau}\right)^{2}+2[1 \\
& -\ln (2)] \Delta \phi \frac{\Delta T}{\tau}-2\left(1-\frac{\pi}{4}\right) \frac{\Delta \beta}{\beta} \frac{\Delta \tau}{\tau}-2\left(1-\frac{\pi}{4}\right) \frac{\Delta T}{\tau} \frac{\Delta \tau}{\tau} \\
& +\frac{\tau}{\theta}\left[\frac{\pi}{4}\left(\frac{\Delta \beta}{\beta}+\frac{\Delta \theta}{\theta}\right)^{2}-4 \ln \left(\frac{\theta}{2 \tau}\right) \Delta \phi \frac{\Delta \theta}{\theta}\right] .
\end{aligned}
$$

At the order $\tau / \theta$ (last block), only the terms containing the $\Delta \theta$ mismatch have been kept since this mismatch does not appear at zero order in $\tau / \theta$. For the other mismatches there are also contributions at first order in $\tau / \theta$ but they can be neglected as compared to the zero-order contributions. The synchronization error can be rewritten as the square root of a positive-definite quadratic form

$$
\sigma=\sqrt{\sum_{i, j=1}^{5} M_{i j} z_{i} z_{j}}=\sqrt{\mathbf{z}^{\mathbf{T}} \mathbf{M z}},
$$

where $\mathbf{z}$ is the five-dimensional vector $(\Delta T / \tau, \Delta \beta / \beta, \Delta \phi, \Delta \theta / \theta, \Delta \tau / \tau)$, while $\mathbf{M}$ is the symmetric characteristic matrix of the quadratic form. Its components $M_{i j}$ can directly be determined from Eq. (68). The diagonal terms of $\mathbf{M}$ correspond to the results we have obtained while considering the mismatches separately, and the nondiagonal terms indicate the various interactions between the different mismatches.

On the other hand, the Wiener-Khintchin formula yields with a satisfying precision the following expression for the cross-correlation function:

$$
\Gamma(s)=\frac{\sin \left(2 \Delta \phi+\frac{s+\Delta T}{\tau}\right)-\sin \left(2 \Delta \phi+\frac{s+\Delta T}{\theta}\right)}{(s+\Delta T)\left(\tau^{-1}-\theta^{-1}\right)} .
$$

As one could have a priori expected, the parameters which individually most influence the cross correlation are still influent.

With these results, one can investigate what the effect of multiple-parameter mismatch is relative to the case of singleparameter mismatches. For this purpose, we make the distinction between two cases: in the first one, only one mismatch can be adjusted while all the others are fixed, while in the second case all the mismatches can simultaneously be tuned.

\section{A. One-parameter optimization}

This particular case of a single tunable parameter is of great experimental importance. For example, in our experimental setup, $\beta$ (which is proportional to the laser output power) and $\phi$ (which is the ratio of two voltages) are much more easily tunable than the length of the delay line or the bandwidth of the filter. Therefore, the point is to find for which value of the tunable mismatch minimizes the synchronization error is reduced.

Let us consider that $z_{k}$ is the tunable mismatch while all the others $z_{i}$ are fixed. In that case, the synchronization error becomes a one-variable function which is minimized when

$$
\frac{\partial \sigma}{\partial z_{k}}=0
$$

that is, for

$$
z_{k}=-\sum_{i=1}^{5} \frac{M_{i k}}{M_{k k}} z_{i}
$$

When some $M_{i k}$ are different from zero, the optimal mismatch is also different from zero. Hence, this equation can be considered as a kind of compensation formula.

Let us take, for example, the case where the master and slave band-pass filters are mismatched. We have shown that the low cutoff mismatch is not influent. Hence, we will focus on the high cut off mismatch $\Delta \tau$, which induces according to Eq. (68) a synchronization error of $\sqrt{1-\pi / 4}|\Delta \tau / \tau|$ when all the other mismatches are uniformly set to 0 . If we tune the $\Delta \beta$ mismatch, we find that synchronization error is minimized when $\Delta \beta$ is given by

$$
\frac{\Delta \beta}{\beta}=\left(1-\frac{\pi}{4}\right) \frac{\Delta \tau}{\tau} .
$$

According to Eq. (68), for this optimal value of $\Delta \beta$ the synchronization error is

$$
\sigma_{\Delta \beta, \Delta \tau}^{\min }=\sqrt{\frac{\pi}{4}\left(1-\frac{\pi}{4}\right)}\left|\frac{\Delta \tau}{\tau}\right|=\sqrt{\frac{\pi}{4}} \sigma_{\Delta \tau},
$$

which is a $12 \%$ reduction in the synchronization error. We therefore reach the quite counterintuitive conclusion that under certain conditions, the multiple-parameter mismatch can improve the quality of the synchronization, since it can lead to a smaller value of $\sigma$. 

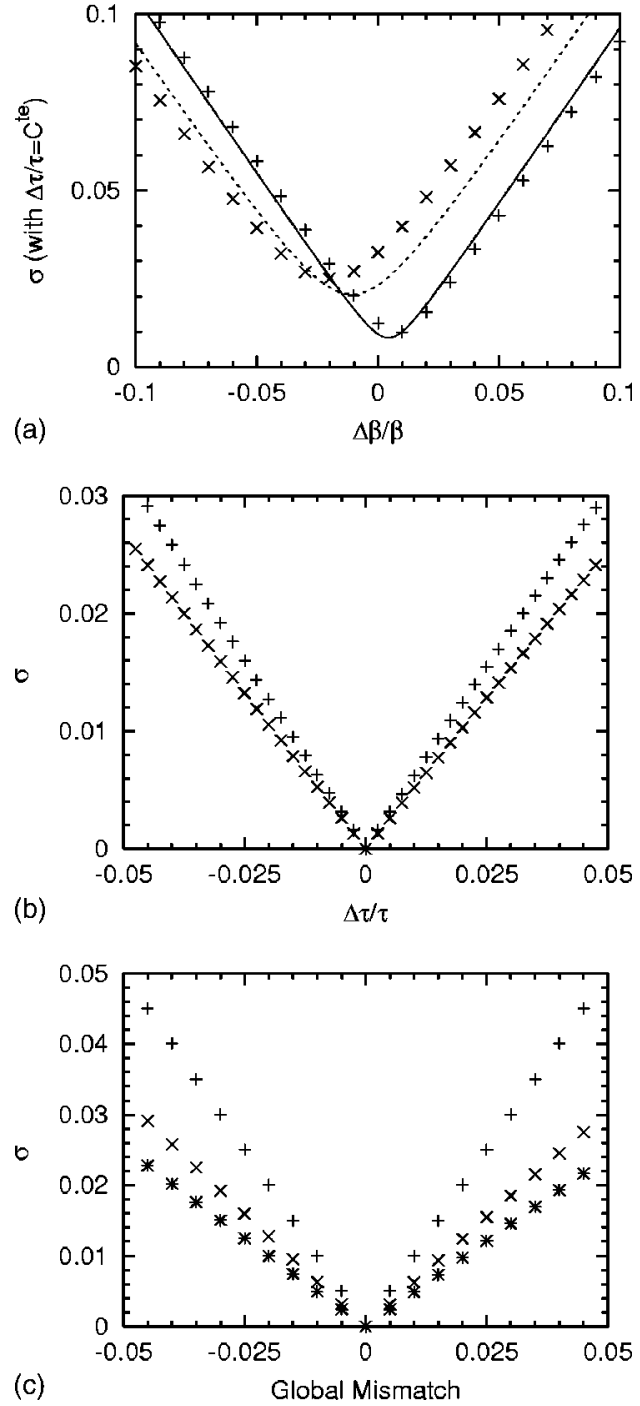

FIG. 8. (a) (top) Synchronization error as a function of $\Delta \beta / \beta$ when the mismatch $\Delta \tau / \tau$ is fixed to $2 \%$ [the solid line shows the analytical approximations and the symbols (+) the numerical results] and $-5 \%$ [dashed line for analytical approximations, $(\times)$ for numerical results]. (b) (center) Results obtained from numerical simulation for the synchronization error as a function of $\Delta \tau / \tau$ when the mismatch $\Delta \beta / \beta=0(+)$ and when $\Delta \beta / \beta$ is optimally tuned accordingly to Eq. (52) (X). (c) (bottom) Evaluation of $\sigma$ from numerical simulation for $\beta$-only mismatch $(+), \tau$-only mismatch $(\times)$, and for a multiple mismatch within the eigendirection (78) determined analytically $(*)$.

This analysis is confirmed by the numerical simulations. In Fig. 8(a), it clearly appears that the minimum error does not occur when $\Delta \beta=0$, but rather when $\Delta \beta$ is shifted by an amount in good concordance with Eq. (73). In Fig. 8(b), the numerical simulation also shows that when the $\Delta \beta$ mismatch is optimally tuned the synchronization error is smaller, by an amount close to the $12 \%$ we have predicted.

It is important to note that even though a off-set phase mismatch $\Delta \phi$ is also easily tunable, it cannot significantly help to compensate for a filter mismatch because $M_{34}$ $\simeq M_{35} \simeq 0$ (however, it can, for example, compensate for a $\Delta T$ mismatch since $M_{31} \neq 0$ ).

\section{B. Multiple-parameter optimization}

Here, we treat the case where all the mismatches can be tuned simultaneously. We are now looking for the optimal manifold in the five-dimensional parameter-mismatch space which leads to the smallest synchronization error growth rate. The first step is to diagonalize $\mathbf{M}$ as

$$
\mathbf{M}=\sum_{k=1}^{5} \Lambda_{k} \mathbf{q}_{\mathbf{k}} \mathbf{q}_{\mathbf{k}}^{\mathbf{T}},
$$

where the $\Lambda_{k}$ are the real and positive eigenvalues of $\mathbf{M}$ and the $\mathbf{q}_{\mathbf{k}}$ are the related orthonormal eigenvectors. Hence, the optimal manifold is the eigendirection corresponding to the smallest eigenvalue $\Lambda^{(-)}$, which is in fact the square of the smallest growth rate. On the other hand, the highest eigenvalue $\Lambda^{(+)}$is related to the eigendirection leading to the strongest error growth rate.

To illustrate this approach, let us take the case of a double and simultaneously tunable $\Delta \beta$ and $\Delta \tau$ mismatch. The corresponding characteristic matrix is

$$
\mathbf{M}=\left(\begin{array}{cc}
1 & -K \\
-K & K
\end{array}\right),
$$

with $K=1-\pi / 4$, and the corresponding eigenvalues are

$$
\Lambda^{( \pm)}=\frac{1}{2}\left[1+K \pm \sqrt{(1+K)^{2}-4 K(1-K)}\right],
$$

that is, $\Lambda^{(-)}=0.160$ and $\Lambda^{(+)}=1.054$. Consequently, the optimal mismatch combination geometrically corresponds to the eigendirection of $\Lambda^{(-)}$, which is defined by

$$
\frac{\Delta \tau}{\tau}=\frac{1-\Lambda^{(-)}}{K} \frac{\Delta \beta}{\beta} .
$$

This mismatch combination leads within that eigendirection to a growth rate of $\sqrt{\Lambda^{(-)}}=0.40$, while this growth rate is 1 for pure $\Delta \beta$ mismatch (60\% reduction of $\sigma$ ), and $\sqrt{1-\pi / 4}$ $=0.46$ for pure $\Delta \tau$ mismatch (14\% reduction). In Fig. 8(c) we show the numerical results for the synchronization error in these three cases. Once again, a combination of mismatches improves the quality of the synchronization. In Fig. 9, the analytical and numerical contour lines are depicted, and illustrate the eigendirection analysis. This kind of contour line patterns have yet been used in Ref. [10] in the case of the wavelength hyperchaos model. It was found numerically that the $\Delta \beta$ and $\Delta \phi$ mismatches were almost "orthogonal," as it is also the case for the electro-optical model considered here since $M_{23}=0$. However, since $\Delta \beta$ and $\Delta \tau$ do precisely interact here at a quadratic approximation $\left(M_{25}\right.$ $\neq 0$ ), the optimal manifold is an oblique line as shown in Fig. 9.

\section{EXPERIMENTAL RESULTS}

For the experimental verification of our theory, the electro-optical modulators were pigtailed $\mathrm{LiNbO}_{3}$ integrated Mach-Zenhder modulators with a dc value of $V_{\pi, d c}=4.0 \mathrm{~V}$, and a rf value (at $1 \mathrm{GHz}$ ) of $V_{\pi, r f}=4.2 \mathrm{~V}$ for $\lambda=1550 \mathrm{~nm}$. 

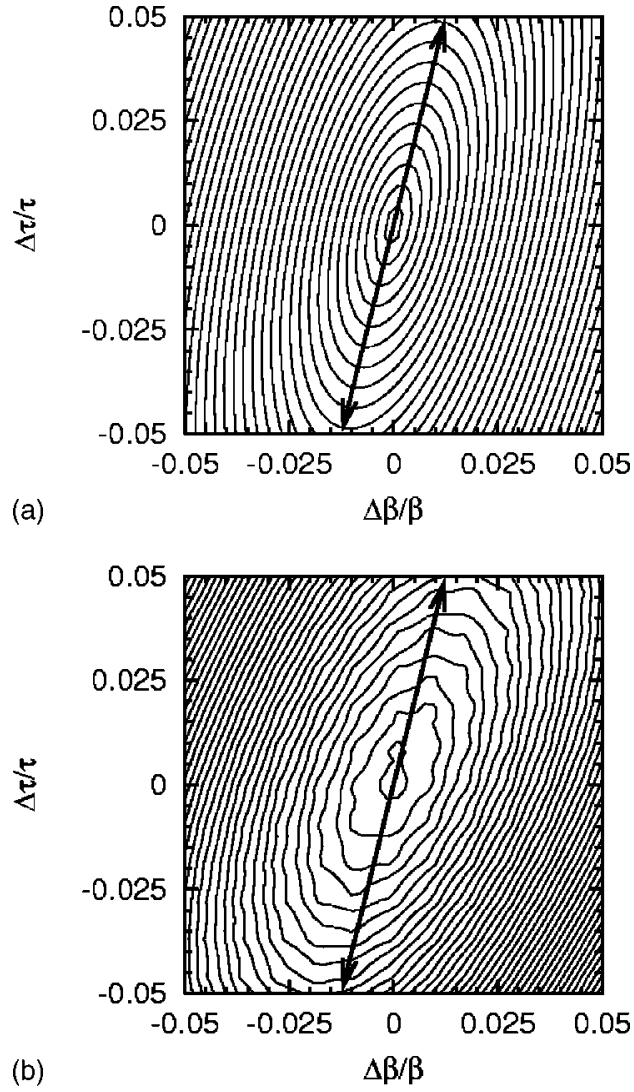

FIG. 9. (a) (top) Analytical level-curve pattern in the parametermismatch subspace of $\Delta \beta / \beta$ and $\Delta \tau / \tau$. The optimal eigendirection which induces the slowest synchronization error growth rate is indicated by the solid double arrow. (b) (bottom) Corresponding numerical level-curve pattern. One can notice that the eigendirection obtained analytically approximately indicates the optimal direction of slowest error growth rate.

One should note that it is the dc value of $V_{\pi}$ that is used in Eq. (3) to define the off-set phase parameter $\phi$. However, only the rf value is relevant for the hyperchaotic generator. The Mach-Zenhder modulators had a electrical bandwidth of $10 \mathrm{GHz}$. The coherent optical feeders of these modulators were $\operatorname{In}_{x} \mathrm{Ga}_{1-x}$ AsP distributed feedback semiconductor lasers with a polarization maintaining pigtail, designed for $10 \mathrm{~Gb} / \mathrm{s}$ digital system equipments. The delay lines were $7 \mathrm{~m}$ long single-mode optical fibers yielding an overall time delay of $30 \mathrm{~ns}$ (taking into account the signal speed reduction in electrical connections). We also used a matched pair of photodetectors with a gain of $2 \mathrm{~V} / \mathrm{mW}$. The amplification within the nonlinear feedback loops was performed by a pair of $\mathrm{rf}$ amplifiers with a power gain of $18 \mathrm{~dB}$ and a bandwidth ranging from $30 \mathrm{kHz}$ to $6.5 \mathrm{GHz}$.

The experimental time traces and Fourier spectrum of the hyperchaotic carrier are depicted in Figs. 10(a) and 10(b), respectively. The bandwidth of the carrier is found to be approximately equal to $7 \mathrm{GHz}$, thereby allowing for chaosencoded communications at bit-rates of several gigabits per second. We have also represented in Fig. 10(c) the transfer functions of the Mach-Zenhder modulators, and one can observe that, experimentally, they are shifted one with respect to the other by an amount of $0.7 \mathrm{~V}$. This shift corresponds to
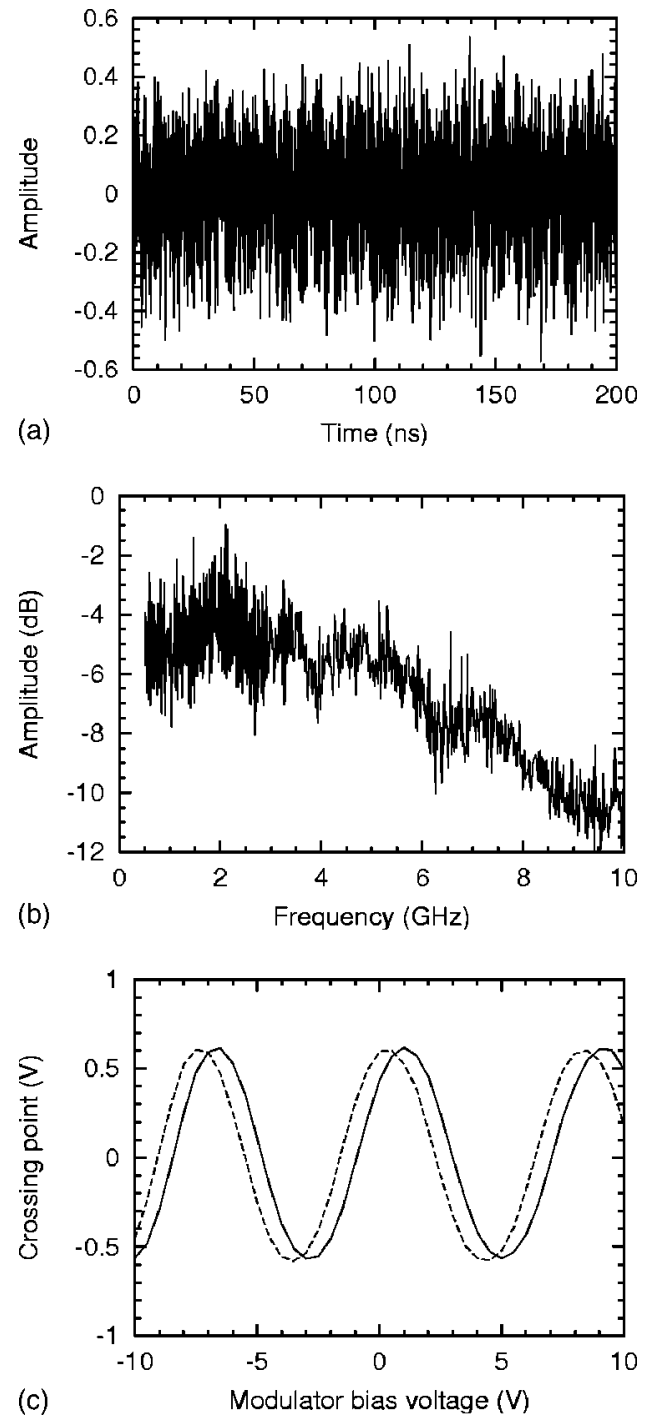

FIG. 10. (a) (top) Experimental hyperchaotic carrier at large scale, with $P=7.60 \mathrm{~mW}$ and $V_{B}=1.05 \mathrm{~V}$ (b) (center) Experimental Fourier spectrum of the hyperchaotic carrier. (c) (bottom) Experimental transfer-function curves for the Mach-Zenhder modulators. Solid line for the emitter and dashed line for the receiver.

the difference between the bias voltages of the two modulators while matching experimentally the $\phi$ parameter for synchronization.

We have first studied the effect of a time-delay mismatch. For that purpose, we have varied the value of $T^{\prime}$ around $T$ using a variable delay line, and the results are presented in Fig. 11. It can be seen that as theoretically predicted, the slave time trace is shifted back and forth according to the value of $\Delta T$. This is of great experimental importance, since in reality, the receiver should synchronize to the emitter irrespective of the coupling delay (or "flying" time) $T_{c}$. Therefore, for this hyperchaotic communication scheme, all the time delays (flying time, time delays due to the electrical connections, and response times of the optoelectronic devices) play exactly the same role as the receiver time delay.

We now focus on the parameters whose mismatch can be easily tuned in our system, namely, the nonlinear feedback 

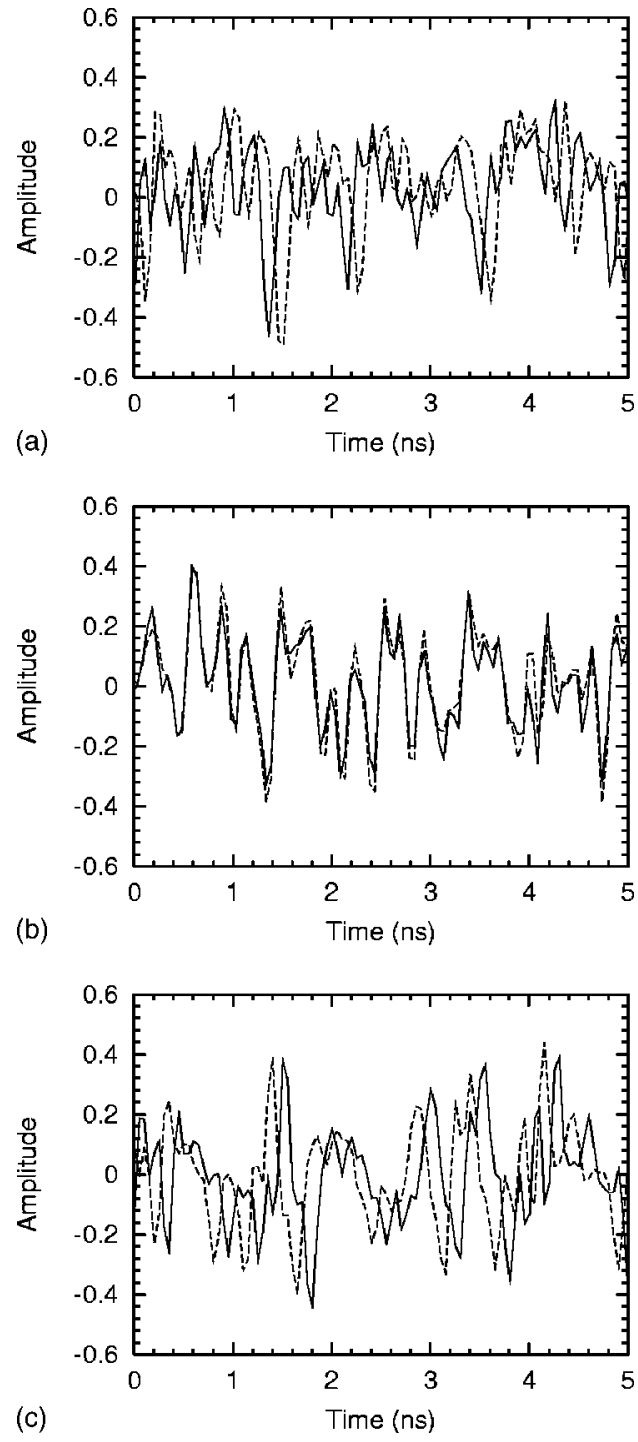

FIG. 11. Experimental chaotic time traces, with $x$ in continuous lines and $y$ in dashed lines. The parameters are $P=7.60 \mathrm{~mW}, V_{B}$ $=1.05 \mathrm{~V}$ (emitter), and $P^{\prime}=3.91 \mathrm{~mW}, V_{B}^{\prime}=0.34 \mathrm{~V}$ (receiver) (a) (top) $\Delta T=0.15 \mathrm{~ns}$, the slave is delayed relatively to the master; (b) (center) $\Delta T=0$, the slave is isochronous to the master; (c) (bottom) $\Delta T=-0.15 \mathrm{~ns}$, the slave anticipates the master.

strength and the off-set phase. Experimentally, $\beta^{\prime}$ and $\phi^{\prime}$ can be tuned through the receiver laser output power $P^{\prime}$ and the receiver modulator bias voltage $V_{B}^{\prime}$, respectively. To achieve our theoretical study, we initially set all the mismatches to 0 , and then we studied their influence one by one. Experimentally, this is obviously impossible. Therefore, the experimental study is intrinsically related to the situation we analyzed in the multiple-mismatches section. The available electronic equipment allowed a matching accuracy down to a few percent, except for the delay which could be tuned within a 2 $\times 10^{-3} \tau$ accuracy. Hence, we principally explored the validity of our theoretical results in the large mismatch case.

Figure 12(a) displays the variations of $\sigma$ as the receiver output power $P^{\prime}$ is increased. Starting from $P^{\prime}=0 \mathrm{~mW}$ (where $\sigma=1$ ), the synchronization error decreases to a minimum (around $P^{\prime}=2 \mathrm{~mW}$ ) and then increases again. In con-
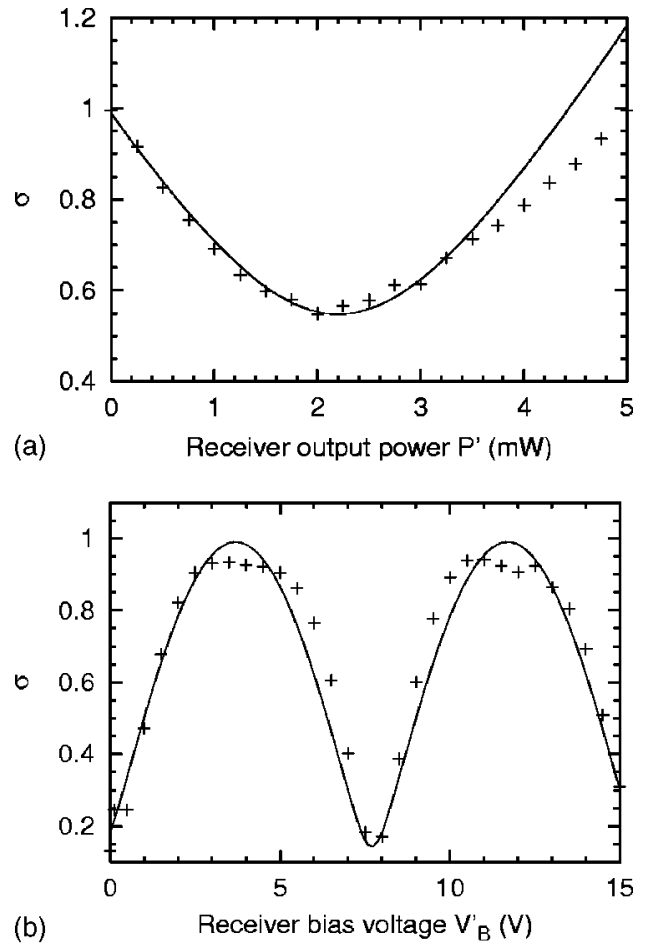

FIG. 12. The symbols show the experimental results of the synchronization error when one parameter is changed while keeping all the others constant. (a) (top) Synchronization error as function of $P^{\prime}$ obtained with $P=2.88 \mathrm{~mW}, V_{B}=1.25 \mathrm{~V}, V_{B}^{\prime}=0.55 \mathrm{~V}$. The solid line corresponds to a function of the form $\sigma=\sqrt{a_{2} P^{\prime 2}+a_{1} P^{\prime}+a_{0}}$. The parameters $a_{i}$ have been fitted to adjust the results. (b) (bottom) synchronization error as function of $V_{B}^{\prime}$ obtained with $P=5.0 \mathrm{~mW}$, $P^{\prime}=2.94 \mathrm{~mW}, V_{B}=3.49 \mathrm{~V}$. The solid line corresponds to a function of the form $\sigma=\sqrt{b_{0}+b_{1} \cos \left(\pi V_{B}^{\prime} / V_{\pi}\right)+b_{2} \sin \left(\pi V_{B}^{\prime} / V_{\pi}\right)}$ predicted theoretically. The parameters $b_{i}$ have been fitted to adjust the results.

cordance with the theory, this experimental curve follows a the square root of a quadratic form (solid line). On the other hand, Fig. 12(b) displays the variations of $\sigma$ as the receiver bias voltage $V_{B}^{\prime}$ is varied. The measured values for $\sigma$ follow the square root of a sinusoidal function in agreement with the theoretical prediction (67). The periodicity is given by the receiver modulator bias voltage and the minima are clearly sharper than the maxima, as theoretically predicted.

As far as the filter mismatches are concerned, it is very difficult to perform any experimental verification. The reason is that the bandwidth of the experimental system results from the combination of the various bandwidths attached to different elements of the electro-optical feedback loop (rf amplifiers, photodetectors, Mach-Zehnder modulator, etc). Therefore, the bandwidth of the experimental setup is neither tunable nor characterized by only two time scales. Hence, even though the fundamental features of the system are captured by the linear first-order band-pass filter, the model has to be theoretically improved at that level.

\section{CONCLUSION}

We have developed an analytical approximation to predict the effect of mismatch for the different parameters of our 
electro-optical system. The predictions give exact results in the case of mismatch in the nonlinear feedback amplitude term, excellent results for the off-set phase mismatch, and good results in the case of the time-delay mismatch and for the mismatch in the filter characteristic time scales. As the receiver is described by a linear differential equation with external forcing, the time-delay mismatch does not distort the receiver time trace, it just shifts it in time. However, if this time shift is not compensated, it becomes the most critical mismatch for the synchronization error. The mismatch $\beta$ can be compensated by rescaling the variables. If this rescaling is not done, the synchronization error grows linearly with the mismatch and a $1 \%$ synchronization error is originated by a $1 \%$ mismatch. We have also found that the highfrequency cutoff of the filter $\tau$ is relatively important $(0.5 \%$ mismatch induces a $1 \%$ synchronization error), while the low cutoff frequency of the filter $\theta$ has a very minor effect on the synchronization error (even a 50\% mismatch induces only about a $0.3 \%$ synchronization error). Finally, the phase mismatch plays also an important role, since a mismatch of about 0.005 rad originates a $1 \%$ synchronization error.

In the case of multiple-parameter mismatch, we have given an analytic insight into a quite counterintuitive phenomenon: multiple-parameter mismatch can sometimes improve the quality of the synchronization. We have also obtained an approximation for the combined effects of all the mismatches taken simultaneously, showing how it is possible to improve the synchronization quality by compensating the mismatch in a given parameter with an added mismatch in another one. In practice, the interest of this multiple mismatch optimization would be at least to identify the conditions under which $\sigma$ does not grow drastically in the case of multiple mismatches between the emitter and the receiver. We have also experimentally verified some of the consequences of our analysis. Particularly, we have verified our theory for the so called easily tunable mismatches, and evidenced the possibility of anticipated, isochronous, and delayed synchronization.
It may be interesting to extend our theory to other laser chaos synchronization schemes. For example, the Fourier spectrum of semiconductor lasers with a short external cavity can also in first approximation be assimilated to a bandlimited white-noise spectrum (the relaxation oscillation frequency of the solitary laser is smaller than the externalcavity-mode spacing frequency, so that the spectrum is not peaky). Therefore, it is a priori possible to apply our results to the study of parameter mismatch for that particular context.

For our own setup, a priority direction for future investigations is the improvement of the synchronization quality at the experimental level. This is by far the most complicated task: for example, even though some of the key components of the materials used for the experiments have been carefully matched at the fabrication level with a very high precision, and even though the experimental time traces evidence a very strong correlation in Fig. 11, the $\sigma$ indicator shows that much is still to be done to attain the final objective of a few percent synchronization error. In that spirit, the high sensibility of these devices functioning at multi-gigahertz frequencies requires to design the emitter and the receiver as integrated circuits. At last, the influence of component and environmental fluctuations are issues that are still to be investigated, both theoretically and experimentally.

\section{ACKNOWLEDGMENTS}

This work has been funded by the European Commission through the research project OCCULT (Contract No. IST2000-29683). Y.C.K and P.C. acknowledge also financial support from the MCyT (Spain) and FEDER under Projects Nos. BFM2000-1108 (CONOCE), BFM2001-0341-C02-02 (SINFIBIO), and TIC2001-4572-E. Y.C.K. also acknowledges financial support from the Direcció General de Recerca, Desenvolupament Técnologic i Innovació of the Govern de les Illes Balears.
[1] See special issue on optical chaos and applications to cryptography, IEEE J. Quantum Electron. 38 (9) (2002), edited by S. Donati and C. R. Mirasso, and references therein.

[2] P. Colet and R. Roy, Opt. Lett. 19, 2056 (1994); C. R. Mirasso, P. Colet, and P. García-Fernandez, IEEE Photonics Technol. Lett. 8, 299 (1996); F. Rogister, A. Locquet, D. Pieroux, M. Sciamanna, O. Deparis, P. Megret, and M. Blondel, Phys. Rev. E 58, 1486 (1998); J.-P. Goedgebuer, L. Larger, and H. Porte, Phys. Rev. Lett. 80, 2249 (1998); G. Van Wiggeren and R. Roy, ibid. 81, 3547 (1998); S. Sivaprakasam and K. A. Shore, Opt. Lett. 24, 1200 (1999); I. Fisher, Y. Liu, and P. Davis, Phys. Rev. A 62, 011801 (2000); H. D. I. Abarbanel, M. B. Kennel, L. Illing, S. Tang, H. F. Chen, and J. M. Liu, IEEE J. Quantum Electron. 37, 1301 (2001); S. Tang and J. M. Liu, Opt. Lett. 26, 1843 (2001); K. Kusumoto and J. Ohtsubo, ibid. 27, 989 (2002); D. Kanakidis, A. Argyris, and D. Syvridis, J. Lightwave Technol. 21, 750 (2003).

[3] V. Annovazzi-Lodi, S. Donati, and A. Scirè, IEEE J. Quantum
Electron. 32, 953 (1996); 33, 1449 (1997); C. Juang, T. M. Hwang, J. Juang, and W.-W. Lin, ibid. 36, 300 (2000); C. R. Mirasso, J. Mulet, and C. Masoller, IEEE Photonics Technol. Lett. 14, 456 (2002).

[4] Y. Chembo Kouomou and P. Woafo, Phys. Rev. E 67, 026214 (2003); Phys. Lett. A 308, 381 (2003); Opt. Commun. 223, 283 (2003).

[5] R. Brown, N. F. Rulkov, and N. B. Tuffilaro, Phys. Rev. E 50, 4488 (1994).

[6] G. A. Johnson, D. J. Mar, T. L. Carroll, and L. M. Pecora, Phys. Rev. Lett. 80, 3956 (1998).

[7] L. M. Pecora and T. L. Carroll, Phys. Rev. Lett. 64, 821 (1990); Phys. Rev. A 44, 2374 (1991); M. A. Matías and J. Güémez, Phys. Lett. A 209, 48 (1995).

[8] A. Sanchez-Díaz, C. R. Mirasso, P. Colet, and P. GarcíaFernandez, IEEE J. Quantum Electron. 35, 292 (1999).

[9] J. Revuelta, C. R. Mirasso, P. Colet, and L. Pesquera, IEEE Photonics Technol. Lett. 14, 140 (2002). 
[10] L. Larger, J.-P. Goedgebuer, and F. Delorme, Phys. Rev. E 57, 6618 (1998).

[11] J.-B. Cuenot, L. Larger, J.-P. Goedgebuer, and W. T. Rhodes, IEEE J. Quantum Electron. 37, 849 (2001).

[12] J.-P. Goedgebuer, P. Levy, L. Larger, C.-C. Chen, and W. T. Rhodes, IEEE J. Quantum Electron. 38, 1178 (2002).

[13] R. Vicente, J. Daudén, P. Colet, and R. Toral, Proc. SPIE
4986, 452 (2003).

[14] B. Dorizzi, B. Grammaticos, M. Le Berre, Y. Pomeau, E. Ressayre, and A. Tallet, Phys. Rev. A 35, 328 (1987).

[15] M. Schwartz, Information, Transmission, Modulation and Noise, 4th ed. (McGraw-Hill, New York, 1990); R. N. McDonough and A. D. Whalen, Detection of Signals in Noise, 2nd ed. (Academic Press, New York, 1995). 